\title{
Effects of Tillage and Cropping Sequences On Crop Production And Environmental Benefits in the North China Plain
}

\author{
Wen-Xuan Liu \\ China Agricultural University \\ Wen-Sheng Liu \\ China Agricultural University \\ Mu-Yu Yang \\ China Agricultural University \\ Yu-Xin Wei \\ China Agricultural University \\ Zhe Chen \\ China Agricultural University \\ Ahmad Latif Virk \\ China Agricultural University \\ Rattan Lal \\ The Ohio State University \\ Xin Zhao ( $\square$ zhaox@cau.edu.cn) \\ China Agricultural University \\ Hai-Lin Zhang \\ China Agricultural University
}

\section{Research Article}

Keywords: Conservation Agriculture, Crop Rotation, Carbon Footprint, Greenhouse Gases Emissions, Carbon Sustainability

Posted Date: January 3rd, 2022

DOI: https://doi.org/10.21203/rs.3.rs-1126359/v1

License: @ (1) This work is licensed under a Creative Commons Attribution 4.0 International License. Read Full License 


\section{Abstract}

The ever-increasing trend of greenhouse gas (GHGs) emissions is accelerating global warming and threatening food security. Environmental benefits and sustainable food production must be pursued locally and globally. Thus, a field experiment was conducted in 2015 to understand how to balance the trade-offs between agronomic productivity and environment quality in the North China Plain (NCP). Eight treatments consisted of two factors, i.e., (i) tillage practices: rotary tillage (RT) and no-till (NT), and (ii) cropping sequences (CS): maize-wheat-soybeanwheat (MWSW), soybean-wheat-maize-wheat (SWMW), soybean-wheat (SW), and maize-wheat (MW). The economic and environmental benefits were evaluated by multiple indicators including the carbon footprint (CF), maize equivalent economic yield (MEEY), energy yield (EY), carbon sustainability index (CSI), etc. Compared with NT, RT increased the EY and MEEY, but emitted 9.4\% higher GHGs. Among different CSs, no significant reduction was observed in CF. The lowest $\left(2.0 \mathrm{Mg} \mathrm{CO}_{2}\right.$-eq ha-1 $\left.\mathrm{yr}^{-1}\right)$ and the highest $\left(5.6 \mathrm{Mg} \mathrm{CO}_{2}\right.$-eq ha-1 $\left.\mathrm{yr}^{-1}\right) \mathrm{CF}$ values were observed under MW and SWMW, respectively. However, CSs with soybean enhanced MEEY and the net revenue due to its higher price compared to that of MW. Although the highest CSI was observed under RT-MW, soybean-based crop rotation could offset the decline in CSI under NT when compared to that for RT. These findings suggest that conservation agriculture (CA) could enhance the balance in trade-offs between economic and environmental benefits. Additional research is needed on how to achieve high crop production by establishing a highly efficient conservation agriculture system in the NCP.

\section{Highlights}

- Conservation agriculture (CA) reduced carbon footprint (CF) by proper nitrogen use.

- Indirect and direct $\mathrm{N}_{2} \mathrm{O}$ emission was the main contributor to CF.

- Soybean-based CA can balance environmental and economic benefits.

- The lower yield under NT partly offsets its environmental benefits.

\section{Introduction}

The anthropogenic climate change is challenging global crop production and human survival (Guo, 2015; IPCC, 2018). Agronomic solutions are needed towards the site-specific variations among different regions, and the discrepancy in food supply-demand makes identification of these solutions urgent and complex challenge. According to the Intergovernmental Panel on Climate Change (IPCC, 2013), agricultural activities emitted almost $20 \%$ of the global greenhouse gases (GHGs). The improper use of farming practices (e.g., overuse of $\mathrm{N}$ fertilizer, high-intensity tillage) are the major direct source of GHGs emissions. In addition, the use of diesel fuel in the process of production, storage, and delivery of agricultural raw materials are indirect source of GHGs emissions (Lal, 2004). Therefore, it is necessary to adopt a set of farming practices with high resource use efficiency, and achieve a win-win strategy to ensure food security and offset GHGs (direct and indirect) emissions.

Best management practices [BMPs, e.g., conservation agriculture] have been identified as effective options to reduce GHGs emissions and maximize economic profits (Janzen et al., 2006; Halvorson et al., 2008). A system-based CA, including minimal tillage, permanent soil cover, and complex crop rotations, has been regarded as one of the systematic solutions for sustainable crop production (Lal, 2015). CA reduces indirect emission of GHGs by decreasing the number of field operations and associated cost inputs (West and Marland, 2002; Lal, 2004). No-till (NT) and crop residue retention (RR), two important principles of CA, can increase soil organic carbon (SOC) sequestration by promoting the formation of soil macro-aggregates and reducing mineralization (Kan et al., 2020a; Kan et al., 2020b; Meng, 2020). However, some long-term studies have indicated that conversion to NT has risks of crop yield reduction during the initial stages of implementation (Huynh et al., 2019). Legume-based crop rotations are recognized as feasible options for nutrient management in CA due to its advantage of biological $\mathrm{N}$ fixation (Zeng, 2018). However, the impacts of legume-based crop rotation on the trade-offs between economic profits and environmental benefits of a CA system has not been well documented. Therefore, a comprehensive assessment of productivity and carbon (C) emission is imperatively helpful to establish an efficient CA system.

Carbon footprint (CF) is a useful tool to assess the environmental impacts of agricultural activities (van der Werf et al., 2013; Schenck and Huizenga, 2014). Additionally, CF based on the scales of different energy yield (EY), maize equivalent economic yield (MEEY), carbon sustainability index (CSI), and net benefits were also functional to determine and compare the $\mathrm{C}$ efficiency from multiple aspects. CF was widely used to evaluate NT, RR, and strategic tillage practices in some previous studies (Xue et al., 2014; Zhang et al., 2016; Liu et al., 2021) and to compare different cropping systems (Yang et al., 2014; Sun et al., 2021). Under CA system, assessing the comprehensive changes in CF, especially from multiple aspects, for the combination of different principles is valuable to optimize CA system through balancing the trade-offs among cereal production, economic, and environmental profits.

The North China Plain (NCP) is one of the major producers of wheat and maize cereals in China. However, the overuse of agricultural inputs such as fertilizers and the ground water in this area has led to severe environmental problems. There are still opportunities to alleviate environmental pressures from agricultural production through the development of cleaner production technologies, such as improved farming 
practices and crop rotation. CA has been adopted in this area since the 1970s (Zhang et al., 2014). Similarly, effects of farming practices on the SOC, yield, economic, and environmental benefits of tillage or crop rotation have been documented for the NCP (Li et al., 2010; Liang et al., 2011; Kan et al., 2020b). Although risks of crop failure under NT have been reported, its environmental benefits and profitability have also been widely reported (Canalli et al., 2020). Nonetheless, the research is lacking for the NCP region with regard to the performance of NT, and how crop rotations interact with it and affect the trade-offs among crop production, economic, and environmental benefits. Thus, it is important to identify the interactions between NT and legume-based crop rotation for establishing an optimized CA system in the NCP. The present study was based on the hypothesis that, in CA system, complex crop rotation, NT, and residue retention promote the balance of economic and environmental tradeoffs, and legume-based crop rotation enhances the net economic benefits. Thus, the objectives of this study were to: 1) evaluate the crop yield, SOC, and CSI under tillage systems combined with different cropping sequences (CSs), and 2) determine a comprehensive assessment of crop yields, economic profits, and environmental costs under CA in this region.

\section{Materials And Methods}

\subsection{Site description}

The field experiment was initiated in 2015 at Wuqiao Experimental Station of China Agricultural University $\left(37^{\circ} 36^{\prime} \mathrm{N}, 116^{\circ} 21^{\prime} \mathrm{E}\right)$ in $\mathrm{Hebei}$ Province. The region is a temperate continental monsoon climate, with an average annual temperature of $13.1^{\circ} \mathrm{C}$ and average annual rainfall of $531 \mathrm{~mm}$. The rainfall is unevenly distributed during the year, and $60-70 \%$ of the annual rainfall is concentrated between June and August. The soil type is Aquic Cambosol of a silt-loam texture (Zhao et al., 2020), containing $11.2 \%$ clay, $18.6 \%$ and, $70.2 \%$ silt. Baseline soil properties in the 0-20 cm layer were $4.97 \mathrm{~g} \mathrm{~kg}^{-1}$ SOC, $0.81 \mathrm{~g} \mathrm{~kg}^{-1} \mathrm{~N}, 44.6 \mathrm{mg} \mathrm{kg}^{-1}$ available P, $92.2 \mathrm{mg} \mathrm{kg}^{-1}$ available $\mathrm{K}$, and $7.51 \mathrm{pH}$.

\subsection{Experimental design}

The field experiment was laid out according to a split-plot design with three replications. The main plots consisted of two tillage practices (started from October 2015), with rotary tillage (RT) and no-till (NT). The subplots consisted of four different CSs (started from October 2017), including maize-wheat-soybean-wheat (MWSW); soybean-wheat-maize-wheat (SWMW); soybean-wheat-soybean-wheat (SW); and maize-wheatmaize-wheat (MW) (Fig. 1). Soil tillage was done only before seeding of wheat, and no tillage was practiced for summer maize and soybeans. Residue management differed between winter wheat and summer maize seasons. Wheat residues were mulched on the soil surface, while maize and soybean residues were chopped into small pieces (5-10 cm long) using a straw shredder. Detailed information on management practices were shown in previous studies (Virk et al., 2021).

\subsection{Soil sampling and analysis}

\subsubsection{Soil organic carbon storage}

Soil samples were obtained at 0-10, 10-20, 20-30, and 30-50 cm depths from each plot near harvesting of each crop (wheat, maize, and soybean) in June and October from 2018 to 2020. After air-drying, soil samples were gently grinded and passed through a 2-mm sieve. The concentration of SOC was determined using a $\mathrm{K}_{2} \mathrm{Cr}_{2} \mathrm{O}_{7}-\mathrm{H}_{2} \mathrm{SO}_{4}$ oxidation procedure (Bao, 2008). To account for differences in soil bulk density, the SOC storage $\left(\mathrm{Mg} \mathrm{ha}^{-1}\right)$ was calculated on equivalent mass basis by using Eq. (1) and (2) (Ellert and Bettany, 1995). The SOC sequestration rate (kg CO ${ }_{2}$-eq $\mathrm{ha}^{-1} \mathrm{yr}^{-1}$ ) was determined by using Eq. (3).

$$
M_{\text {soil }, i}=B D_{i} \times \mathrm{T}_{i} \times 1000
$$

1

$$
\text { SOCstorage }=\sum_{i=1}^{n}\left[M_{\text {soil }, i} \times \operatorname{Conc}_{i}+\left(M_{o, i}-M_{\text {soil }, i}\right) \times \text { Conc }_{i+1}\right] \times 0.001
$$

2

$$
\text { SOCsequestrationrate }=\frac{S S 2020-S S 2015}{4} \times \frac{44}{12}
$$

3

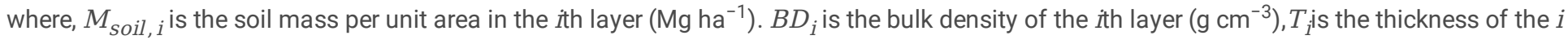
th layer (m). Conc $_{i}$ is the concentration of SOC in ith layer. Conc $_{i+1}$ is the concentration of SOC in $i+7$ th layer. $M_{O, i}$ is the designated equivalent mass of each layer (i.e., the maximum soil mass), $i$ is the soil depth interval,a, $\mathrm{d} i=1,2,3$ and 4 for 0-10, 10-20, 20-30 cm, and 30-50 cm layer, respectively. The number of 1000 and 0.001 are unit conversion coefficients. SS2015 and SS2020 are the SOC storage at the beginning of the 
experiment and the SOC storage at the harvest of winter wheat in 2020, respectively, and 4 represents the duration of the experiment. The $44 / 12$ is the coefficient that converts $\mathrm{C}$ to $\mathrm{CO}_{2}$.

\subsubsection{Crop residues and carbon inputs from crop resides}

Crops grain yield were measured in a quadrant of $2 \mathrm{~m}^{2}$ for winter wheat and summer soybean in each plot, and double rows of $10 \mathrm{~m}$ for summer maize in each plot. The amount of crop residue was calculated as the ratios of grain: straw and root: straw (Zhao et al., 2018).

The $\mathrm{C}$ input included aboveground residues and belowground roots. The $\mathrm{C}$ input of the aboveground residues was calculated by using Eq. (4), and that of the belowground C input by Eq. (5) (Zhao et al., 2018).

$$
C_{a}=G Y \times \frac{1-W C}{G S \text { ratio }} \times 0.45
$$

4

$$
C_{b}=G Y \times \frac{1-W C}{G S \text { ratio }} \times R S \text { ratio } \times 0.45
$$

5 where, GY represents the grain yield of each season. WC represents the water content of the grain, which is $0.125,0.135$, and 0.103 for wheat, maize, and soybean, respectively. GSratiorepresents grain: straw, and wheat, maize, and soybean are $0.846,0.955$, and 0.524 , respectively. $R S$ ratio represents root: straw, and wheat, maize, and soybean are $0.2,0.28$, and 0.196 , respectively. The conversion coefficient for crop biomass to $\mathrm{C}$ content is 0.45 (Fang et al., 2007).

\subsubsection{Net revenue}

Net revenue was calculated based on the economic value from grain minus total inputs during crop production. The prices of all grain and inputs were calculated according to the actual local conditions (Liu et al., 2021). The total inputs involved farming, harvesting, fertilizer, herbicide, and irrigation. The economic profit was calculated by Eq. (6) (Ray et al., 2016).

$$
\text { Benefit }: \text { cost ratio }=\frac{\text { Grain income }}{\text { Total input }}
$$

6

\subsubsection{Carbon footprints}

International Organization for Standardization (ISO) has defined the CF as the sum of GHGs directly and indirectly produced by crops during a single life cycle and can be expressed in carbon dioxide equivalents ( $\mathrm{CO}_{2}$-eq) $(\mathrm{ISO}, 2013)$. CF consisted mainly of off-farm input production and on-farm application. The system boundary was from sowing (including seedbed preparation) to the harvesting of crops. The involved inputs, practices, or inventories are shown in Fig. 2.

The $\mathrm{N}$ content in aboveground crop residues was calculated with Eq. (7). $\mathrm{N}_{2} \mathrm{O}$ emission in this study was calculated by using inputs of $\mathrm{N}$ fertilizer, crop residues, and emission factors. Therefore, the direct $\mathrm{N}_{2} \mathrm{O}$ emission was calculated by using Eq. (8), and indirect $\mathrm{N}_{2} \mathrm{O}$ emission by Eq. (9). The total emission of $\mathrm{N}_{2} \mathrm{O}$ was calculated by Eq. (10).

$$
F_{S}=\left(C_{S i}+C_{r i}\right) \times N_{C(i)}
$$

7

$$
N_{2} O_{D E}=\left(F_{S}+F_{N}\right) \times \sigma_{1} \times \frac{44}{28}
$$

8

$$
N_{2} O_{I E}=\left[F_{N} \times F R A C_{G A S F} \times \sigma_{2}+\left(F_{S}+F_{N}\right) \times F R A C_{L E A C H} \times \sigma_{3}\right] \times \frac{44}{28}
$$

$$
G H G_{N_{2} O}=\left(N_{2} O_{D E}+N_{2} O_{I E}\right) \times 298
$$


where, $F_{S}$ and $F_{N}$ are the $\mathrm{N}$ content of aboveground crop residues and $\mathrm{N}$ fertilizer application. $N_{C(i)}$ is the $\mathrm{N}$ content of different crops. $\sigma_{1}, \sigma_{2}$, and $\sigma_{3}$ are the emission coefficients of $\mathrm{N}$ inputs, volatilization of $\mathrm{N}$ fertilizer, and $\mathrm{N}$ leaching, respectively. $F R A C_{G A S F}$ represents the fraction of $\mathrm{N}$ fertilizer volatilized $\left(\mathrm{NH}_{3}\right.$ and $\left.\mathrm{NO}_{\mathrm{X}}-\mathrm{N}\right)$. FRAC $L E A C H$ represents the fraction of $\mathrm{N}$ leaching. The value of $44 / 28$ and 298 are the coefficients of $\mathrm{N}_{2}$ to $\mathrm{N}_{2} \mathrm{O}$ and $\mathrm{N}_{2} \mathrm{O}$ to $\mathrm{CO}_{2}$-eq, respectively (Table 1 ).

Table 1

GHGs emissions coefficients for different agricultural material inputs.

\begin{tabular}{|c|c|c|c|c|}
\hline Item & Abbreviation & $\begin{array}{l}\text { Emission } \\
\text { factor }\end{array}$ & Unit & Source \\
\hline Tillage & $\mathrm{EF}_{\mathrm{T}}$ & 3.37 & $\mathrm{~kg} \mathrm{CO}_{2}$-eq kg ${ }^{-1}$ & Zhang et al. (2013) \\
\hline Harvest & $\mathrm{EF}_{\mathrm{H}}$ & 3.37 & $\mathrm{~kg} \mathrm{CO}_{2}$-eq kg ${ }^{-1}$ & Zhang et al. (2013) \\
\hline Nitrogenous fertilizer & $\mathrm{EF}_{\mathrm{N}}$ & 3.44 & $\mathrm{~kg} \mathrm{CO}_{2}$-eq kg ${ }^{-1}$ & Chen et al. (2015) \\
\hline Phosphatic fertilizer & $\mathrm{EF}_{\mathrm{P}}$ & 0.4 & $\mathrm{~kg} \mathrm{CO}_{2}$-eq kg ${ }^{-1}$ & Chen et al. (2015) \\
\hline Potassic fertilizer & $\mathrm{EF}_{\mathrm{K}}$ & 0.3 & $\mathrm{~kg} \mathrm{CO}_{2}$-eq kg ${ }^{-1}$ & Chen et al. (2015) \\
\hline Herbicides & $\mathrm{EF}_{2 \mathrm{H}}$ & 18 & $\mathrm{~kg} \mathrm{CO}_{2}$-eq kg ${ }^{-1}$ & Yang et al. (2014) \\
\hline Irrigation consumptions & $\mathrm{EF}_{\mathrm{I}}$ & 1.12 & $\mathrm{CO}_{2}-\mathrm{eq} \cdot \mathrm{kW} \cdot \mathrm{h}^{-1}$ & Zhang et al. (2013) \\
\hline $\mathrm{N}$ fertilizer volatilization fraction & FRAC $_{\text {GASF }}$ & 0.1 & $\begin{array}{l}\mathrm{Kg} \mathrm{NH}_{3}-\mathrm{N}+\mathrm{NO}_{X}-\mathrm{N} \text { volatilized } \mathrm{kg}^{-1} \\
\mathrm{~N} \text { input }\end{array}$ & IPCC, (2006) \\
\hline $\mathrm{N}$ leaching fraction & FRAC $_{\text {LEACH }}$ & 0.3 & $\mathrm{~kg} \mathrm{~N} \mathrm{~kg}^{-1} \mathrm{~N}$ input & IPCC, (2006) \\
\hline $\begin{array}{l}\text { Direct } \mathrm{N}_{2} \mathrm{O} \text { emission from } \mathrm{N} \text { fertilizer on } \\
\text { upland crops }\end{array}$ & $\sigma_{1}$ & 0.01 & $\mathrm{~kg} \mathrm{~N}_{2} \mathrm{O}-\mathrm{N} \mathrm{kg}^{-1} \mathrm{~N}$ input & IPCC, (2006) \\
\hline $\begin{array}{l}\text { Indirect } \mathrm{N}_{2} \mathrm{O} \text { emission from } \mathrm{N} \text { fertilizer } \\
\text { volatilization }\end{array}$ & $\sigma_{2}$ & 0.01 & $\begin{array}{l}\mathrm{kg} \mathrm{N}_{2} \mathrm{O}-\mathrm{N} \mathrm{kg}^{-1} \mathrm{NH}_{3}-\mathrm{N}+\mathrm{NO}_{X}-\mathrm{N} \\
\text { volatilized }\end{array}$ & IPCC, (2006) \\
\hline $\begin{array}{l}\text { Indirect } \mathrm{N}_{2} \mathrm{O} \text { emission from } \mathrm{N} \text { fertilizer } \\
\text { leaching }\end{array}$ & $\sigma_{3}$ & 0.0075 & $\mathrm{~kg} \mathrm{~N}_{2} \mathrm{O}-\mathrm{N} \mathrm{kg}^{-1} \mathrm{~N}$ leaching & IPCC, (2006) \\
\hline $\mathrm{N}$ content of winter wheat & $\mathrm{N}_{\mathrm{CW}}$ & 0.0052 & $\mathrm{~kg} \mathrm{~N}(\mathrm{~kg} \text { dry matter })^{-1}$ & NDRC (2011) \\
\hline $\mathrm{N}$ content of summer maize & $\mathrm{N}_{\mathrm{CM}}$ & 0.0058 & $\mathrm{~kg} \mathrm{~N}(\mathrm{~kg} \text { dry matter })^{-1}$ & NDRC (2011) \\
\hline $\mathrm{N}$ content of summer soybean & $\mathrm{N}_{\mathrm{CS}}$ & 0.018 & $\mathrm{~kg} \mathrm{~N}(\mathrm{~kg} \text { dry matter })^{-1}$ & NDRC (2011) \\
\hline Winter wheat seed & & 0.58 & $\mathrm{~kg} \mathrm{CO}_{2}$-eq kg ${ }^{-1}$ & Ecoinvent 2.2 \\
\hline Summer maize seed & & 1.93 & $\mathrm{~kg} \mathrm{CO}_{2}$-eq kg ${ }^{-1}$ & Ecoinvent 2.2 \\
\hline Summer soybean seed & & 0.92 & $\mathrm{~kg} \mathrm{CO}_{2}$-eq kg ${ }^{-1}$ & $\begin{array}{l}\text { West and Marland } \\
(2002)\end{array}$ \\
\hline
\end{tabular}

$\mathrm{CO}_{2}$ emissions from agricultural inputs (i.e., seeds, chemical fertilizers, herbicides, and diesel fuel produced by farming, harvesting, and irrigation) were calculated by Eq. (11) (Lal, 2004).

$$
G W P_{\text {input }}=\sum_{i}^{n} A I_{i} \times E F_{i}
$$

11 where, $A I$ represents the amount of each item of input (Table 2). EFrepresents its emission coefficient (Table 1). represents different agricultural inputs. 
Table 2

Mean agricultural inputs of different tillage practices and cropping sequences.

\begin{tabular}{|c|c|c|c|c|c|c|c|c|c|c|}
\hline Item & Treatment & & $\begin{array}{l}\text { Seed } \\
(\mathrm{kg} \\
\left.\mathrm{ha}^{-1}\right)\end{array}$ & $\begin{array}{l}\text { Tillage }^{\mathrm{a}} \\
(\mathrm{kg} \\
\left.\mathrm{ha}^{-1}\right)\end{array}$ & $\begin{array}{l}\text { Harvest }^{\mathrm{a}} \\
(\mathrm{kg} \\
\left.\mathrm{ha}^{-1}\right)\end{array}$ & $\begin{array}{l}\text { Nitrogenous } \\
\text { fertilizer } \\
\left(\mathrm{kg} \mathrm{ha}^{-1}\right)\end{array}$ & $\begin{array}{l}\text { Phosphatic } \\
\text { fertilizer } \\
\left(\mathrm{kg} \mathrm{ha}^{-1}\right)\end{array}$ & $\begin{array}{l}\text { Potassic } \\
\text { fertilizer } \\
\left(\mathrm{kg}^{-1}\right) \\
\left.\mathrm{ha}^{-1}\right)\end{array}$ & $\begin{array}{l}\text { Herbicides }^{\mathrm{b}} \\
\left(\mathrm{kg} \mathrm{ha}^{-1}\right)\end{array}$ & $\begin{array}{l}\text { Irrigation } \\
\text { Consumptions } \\
\left(\mathrm{kWh} \mathrm{ha}^{-1}\right)\end{array}$ \\
\hline \multirow{2}{*}{$\begin{array}{l}\text { Winter } \\
\text { wheat }\end{array}$} & RT & & 300 & 69.125 & 42.5 & 318 & 103.5 & 107 & 0.225 & 675 \\
\hline & NT & & 300 & 62 & 42.5 & 318 & 103.5 & 107 & 0.225 & 675 \\
\hline \multirow{2}{*}{$\begin{array}{l}\text { Summer } \\
\text { maize }\end{array}$} & RT & & 60 & 13.5 & 38.5 & 339.75 & 130.435 & 120 & 1.5 & 675 \\
\hline & NT & & 60 & 13.5 & 38.5 & 339.75 & 130.435 & 120 & 1.5 & 675 \\
\hline \multirow{2}{*}{$\begin{array}{l}\text { Summer } \\
\text { soybean }\end{array}$} & RT & & 150 & 13.5 & 38.5 & 183.75 & 130.435 & 120 & 0 & 675 \\
\hline & NT & & 150 & 13.5 & 38.5 & 183.75 & 130.435 & 120 & 0 & 675 \\
\hline $\begin{array}{l}\text { The } \\
\text { entire }\end{array}$ & RT & MWSW & 405 & 82.625 & 81 & 579.75 & 233.935 & 227 & 0.975 & 1350 \\
\hline \multirow{7}{*}{$\begin{array}{l}\text { growing } \\
\text { season }\end{array}$} & & SWMW & 405 & 82.625 & 81 & 579.75 & 233.935 & 227 & 0.975 & 1350 \\
\hline & & SW & 450 & 82.625 & 81 & 501.75 & 233.935 & 227 & 0.225 & 1350 \\
\hline & & MW & 360 & 82.625 & 81 & 657.75 & 233.935 & 227 & 1.725 & 1350 \\
\hline & NT & MWSW & 405 & 75.5 & 81 & 579.75 & 233.935 & 227 & 0.975 & 1350 \\
\hline & & SWMW & 405 & 75.5 & 81 & 579.75 & 233.935 & 227 & 0.975 & 1350 \\
\hline & & SW & 450 & 75.5 & 81 & 501.75 & 233.935 & 227 & 0.225 & 1350 \\
\hline & & MW & 360 & 75.5 & 81 & 657.75 & 233.935 & 227 & 1.725 & 1350 \\
\hline \multicolumn{11}{|c|}{$\begin{array}{l}\text { RT: rotary tillage; NT: no-till. MWSW: maize-wheat-soybean-wheat; SWMW: soybean-wheat-maize-wheat; SW: soybean-wheat; and MW: } \\
\text { maize-wheat. }\end{array}$} \\
\hline \multicolumn{11}{|c|}{ a Diesel consumption at tillage and harvest was calculated by multiplying the actual amount applied by the diesel density. } \\
\hline
\end{tabular}

CF per unit of area $\left(\mathrm{CF}_{\mathrm{A}}\right)\left(\mathrm{kg} \mathrm{CO}_{2}\right.$-eq ha-1 $\left.\mathrm{yr}^{-1}\right)$ was calculated by Eq. (12).

$$
C F_{A}=G W P_{\text {input }}+G H G_{N_{2} 0}+\Delta S O C
$$

12

where, is the mean annual $\mathrm{C}$ change in $0-30 \mathrm{~cm}$ soil layer.

To unify the average annual crop yield for crops, the actual yield was converted into $\mathrm{EY}$ and $\mathrm{MEEY}$. The $\mathrm{CF}$ per $\mathrm{kg}$ of EY $\left(\mathrm{CF}_{\mathrm{EY}}\right)\left(\mathrm{kg} \mathrm{CO}_{2}\right.$-eq $\mathrm{GJ}^{-1}$ $\mathrm{yr}^{-1}$ ) was calculated by Eq. (13) and (14).

$$
E_{Y}=\left(Y_{g} \times E_{g}+Y_{s} \times E_{s}\right) / 10^{3}
$$

13

$$
C F_{E Y}=\frac{C F_{A}}{E Y}
$$

14

where, $E_{Y}\left(\mathrm{GJ}\right.$ ha $\left.{ }^{-1}\right)$ represents energy yield. $Y_{g}$ and $Y_{s}$ are the yield of grain and straw. $E_{g}$ and $E_{s}$ are the calorific value of yield and straw. The grain calorific values of wheat, maize, and soybean were $16.3,16.3$, and $20.9 \mathrm{MJ} \mathrm{kg}^{-1}$, respectively. The straw calorific values of wheat, maize, and soybean were 14.6, 14.6, and $15.1 \mathrm{MJ} \mathrm{kg}^{-1}$, respectively (Chen, 2002). 
The CF per kg of MEEY (CF MEEY, $\mathrm{kg} \mathrm{CO}_{2}$-eq kg ${ }^{-1} \mathrm{yr}^{-1}$ ) was calculated by Eq. (15) and (16) (Pradhan et al., 2018).

$$
M E E Y=Y_{i} \times \frac{P_{i}}{P_{M}}
$$

15

$$
C F_{M E E Y}=\frac{C F_{A}}{M E E Y}
$$

16

where, $\left(\mathrm{kg} \mathrm{ha}^{-1}\right)$ is the yield, ( US $\$ \mathrm{~kg}^{-1}$ ) is the price, is wheat and soybean. The local prices of wheat, maize, and soybean are $0.38,0.3$, and 0.88 US $\$ \mathrm{~kg}^{-1}$, respectively.

The CF per unit of economic revenue $\left(\mathrm{CF}_{\mathrm{E}}\right)\left(\mathrm{kg} \mathrm{CO}_{2}-\mathrm{eq}^{-1} \mathrm{~S}^{-1} \mathrm{yr}^{-1}\right)$ was calculated by Eq. (17).

$$
C F_{E}=\frac{C F_{A}}{E}
$$

17

where, $\mathrm{E}$ is the total revenue (US \$ ha-1) (Table 4).

\begin{tabular}{|c|c|c|c|c|c|}
\hline \multicolumn{2}{|c|}{ Treatment } & \multirow{2}{*}{$\begin{array}{l}\text { Production cost } \\
\text { (US \$ ha-1) } \\
1485.29\end{array}$} & \multirow{2}{*}{$\begin{array}{l}\text { Yield revenue } \\
\text { (US \$ ha-1) } \\
4974.95\end{array}$} & \multirow{2}{*}{$\begin{array}{l}\text { Net revenue } \\
\left(\text { US } \$ \text { ha }^{-1}\right) \\
3489.65\end{array}$} & \multirow{2}{*}{$\begin{array}{l}\text { input output economic ratio } \\
\left.\text { (US } \$ \mathrm{ha}^{-1}\right) \\
3.3\end{array}$} \\
\hline RT & MWSW & & & & \\
\hline & SWMW & 1367.29 & 4642.20 & 3274.90 & 3.4 \\
\hline & SW & 1485.29 & 5342.80 & 3857.52 & 3.6 \\
\hline & MW & 1249.29 & 4680.48 & 3431.18 & 3.7 \\
\hline \multirow[t]{4}{*}{ NT } & MWSW & 1358.81 & 4486.45 & 3127.64 & 3.3 \\
\hline & SWMW & 1358.81 & 4083.91 & 2725.10 & 3.0 \\
\hline & SW & 1524.36 & 4372.78 & 2848.43 & 2.9 \\
\hline & MW & 1240.83 & 3800.31 & 2559.49 & 3.1 \\
\hline
\end{tabular}

Table 4

Comparison of annual average economic profits (\$ value can be rounded up to the whole \$ and delete cents).

\subsubsection{Carbon sustainability index (CSI)}

The carbon sustainability index (CSI) was calculated by Eq. (18) (Lal, 2004).

$$
C S I=\frac{C_{\text {output }}-C_{\text {emission }}}{C_{\text {emission }}}
$$

18

where, $C_{\text {output }}$ is the average $\mathrm{C}$ produced by biomass, which includes grain, straw, and root. The average $\mathrm{C}$ produced by straw and root was calculated in Eq. (4) and (5), respectively. $C_{\text {emission }}$ was calculated by Eq. (12).

\subsection{Data analysis}

IBM SPSS version 23.0 software was used to perform the analysis of variance (ANOVA). The means and interactions comparisons were analyzed using the least significant difference (LSD) test. The average values of the selected CF-related parameters were standardized to show their performance through radar diagrams (Ladha et al., 2016). Origin Pro 2017 was used to design figures.

\section{Results}




\subsection{Grain yield}

EY was significantly affected by tillage practices and CSs $(P<0.05$, Table 3$)$, while a non-significant interaction was observed between tillage practices and CSs. RT-MW and NT-SW were observed with the highest and lowest EY, respectively. Among different CSs under RT, the EY of MWSW, SWMW, and SW were 15.2\%, 17.2\%, and 19.5\% lower than that of MW, respectively. Similarly, the EY of MWSW, SWMW, and MW were $25.7 \%, 12.8 \%$, and $28.7 \%$ higher than that of SW under NT, respectively. Similarly, tillage and CSs were observed to affect MEEY individually $(P<0.05)$, but with no significant interaction (Table 3). Under RT, SW and SWMW were observed to have the highest and lowest MEEY. Under NT, the highest MEEY was observed under MWSW, which was higher than those under MWSW, SW, and MW by $9.9 \%$, $2.6 \%$, and $18.1 \%$, respectively.

Table 3

Annual average energy and maize equivalent economic yield of different tillage practices and cropping sequences.

\begin{tabular}{|c|c|c|c|}
\hline \multicolumn{2}{|c|}{ Treatment } & \multirow{2}{*}{$\begin{array}{l}\text { Energy yield } \\
\left(\text { GJ ha }^{-1}\right) \\
382.04 \mathrm{~b}\end{array}$} & \multirow{2}{*}{$\begin{array}{l}\text { Maize equivalent economic yield }\left(\mathrm{kg} \mathrm{ha}^{-1}\right) \\
15102.68 \mathrm{~b}\end{array}$} \\
\hline RT & MWSW & & \\
\hline & SWMW & 373.12 bc & $14831.30 \mathrm{bc}$ \\
\hline & SW & 362.86 bc & $17069.66 \mathrm{a}$ \\
\hline & MW & $450.74 \mathrm{a}$ & 14953.62 b \\
\hline \multirow[t]{4}{*}{ NT } & MWSW & 361.38 bc & $14333.71 \mathrm{bc}$ \\
\hline & SWMW & $324.17 \mathrm{~cd}$ & $13047.66 \mathrm{~cd}$ \\
\hline & SW & $287.39 \mathrm{~d}$ & $13970.53 \mathrm{bc}$ \\
\hline & MW & 369.83 bc & $12141.57 \mathrm{~d}$ \\
\hline $\mathrm{T}$ & & $<0.05$ & $<0.05$ \\
\hline CS & & $<0.05$ & $<0.05$ \\
\hline $\mathrm{T} \times \mathrm{CS}$ & & 0.207 & 0.193 \\
\hline
\end{tabular}

\subsection{Net revenue}

The net revenue of each CS was higher under RT than that under NT (Table 4), that is $11.6 \%, 20.2 \%, 35.4 \%$, and $34.1 \%$ higher under RT than that under NT for MWSW, SWMW, SW, and MW, respectively (Table 4). RT-SW and NT-MW were observed with the highest and lowest net revenue, respectively. RT-SW was 35.4\% higher than that under NT-SW, and NT-MW was $25.4 \%$ lower than that under RT-MW. Among RT, SW (the highest) was $17.8 \%$ higher than that under SWMW (the lowest). Among NT, MWSW (the lowest) was $22.2 \%$ higher than that under MW (the lowest).

Similarly, input output economic ratios were higher under RT than that under NT for the four CSs (Table 4). However, the input output economic ratio of MWSW was almost the same under RT and NT. RT-MW and NT-SW were observed with the highest and lowest input: output economic ratio, respectively. The input: output economic ratio under RT-MW was 12.1\%, 8.8\%, and 2.8\% higher than that under MWSW, SWMW, and SW, respectively. The highest input: output economic ratio under NT was observed for MWSW, which was 10.0\%, 13.0\%, 8.0\%, and 6.5\% higher than that under SWMW, SW, and MW, respectively.

\subsection{SOC storage}

Tillage and CSs were observed to affect SOC storage and SOC sequestration rate at 0-30 cm depth individually ( $P<0.05$, Fig. 3), but without significant interaction between tillage and CSs. The highest SOC storage and SOC sequestration rate were both observed under RT-MW. The SOC storage was $9.4 \%, 13.5 \%, 11.1 \%, 24.5 \%, 25.3 \%, 13.9 \%, 13.4 \%$ higher under RT-MW than that under RT-MWSW, RT-SWMW, RT-SW, NT-MWSW, NTSWMW, NT-SW and NT-MW, respectively. SOC sequestration rate was 9.8\%, 15.1\%, 21.3\%, 47.2\%, 42.4\%, 17.7\%, 14.0\% higher under RT-MW than that under RT-MWSW, RT-SWMW, RT-SW, NT-MWSW, NT-SWMW, NT-SW and NT-MW, respectively. For different CSs under RT, SWMW (the lowest) decreased SOC storage by $11.9 \%$ compared to MW (the highest). Similarly, among different CSs under NT, SWMW (the lowest) decreased SOC storage by $8.9 \%$ compared to MW (the highest). Furthermore, among different CSs under RT, MW increased SOC sequestration rate by $21.3 \%$ compared to SW (the lowest). Among different CSs under NT, MW increased SOC sequestration rate by $29.1 \%$ compared to SWMW (the lowest).

\subsection{Carbon footprints}


Significant differences in $\mathrm{CF}_{\mathrm{A}}$ were observed among treatments $\left(P<0.05\right.$, Table 5). The highest $\mathrm{CF}_{\mathrm{A}}$ was observed under NT-SWMW (5584.49 kg $\mathrm{CO}_{2}$-eq ha ${ }^{-1} \mathrm{yr}^{-1}$ ), which was 49.9\%, 42.9\%, 12.3\%, 64.3\%, 14.2\%, 40.4\%, and 34.3\% higher than that under RT-MWSW, RT-SWMW, RT-SW, RT-MW (the lowest), NT-MWSW, NT-SW, and NT-MW respectively. The highest $\mathrm{CF}_{\mathrm{EY}}$ was observed under NT-SWMW $\left(17.23 \mathrm{~kg} \mathrm{CO}_{2}\right.$-eq GJ $\left.^{-1} \mathrm{yr}^{-1}\right)$, with the lowest under RT-MW (4.42 $\mathrm{kg} \mathrm{CO}_{2}$-eq $\mathrm{GJ}^{-1} \mathrm{yr}^{-1}$ ), respectively (Table 5). Significant differences in $\mathrm{CF}_{\mathrm{MEEY}}$ were observed among treatments. NTSWMW (the highest, $0.43 \mathrm{~kg} \mathrm{CO}_{2}$-eq kg ${ }^{-1} \mathrm{yr}^{-1}$ ) increased $\mathrm{CF}_{\mathrm{MEEY}}$ by $28.0-68.9 \%$ compared to the other treatments. The lowest $\mathrm{CF}_{\mathrm{MEEY}}$ was observed under RT-MW (0.13 $\mathrm{kg} \mathrm{CO}_{2}$-eq $\left.\mathrm{kg}^{-1} \mathrm{yr}^{-1}\right)$. NT-SWMW was the highest with $\mathrm{CF}_{\mathrm{E}}$ (Table 5), which was $113.3 \%$ higher than that under RTSWMW (the lowest).

Table 5

Average carbon footprint of different tillage practices and cropping sequences.

\begin{tabular}{|c|c|c|c|c|c|}
\hline \multicolumn{2}{|c|}{ Treatment } & \multirow{2}{*}{$\begin{array}{l}\mathrm{CF}_{\mathrm{A}} \\
\left(\mathrm{kg} \mathrm{CO}_{2} \text { eq ha-1 } \mathrm{yr}^{-1}\right)\end{array}$} & \multirow{2}{*}{$\begin{array}{l}\mathrm{CF}_{\mathrm{EY}} \\
\left(\mathrm{kg} \mathrm{CO}_{2} \text { eq } \mathrm{GJ}^{-1} \mathrm{yr}^{-1}\right)\end{array}$} & \multirow{2}{*}{$\begin{array}{l}\mathrm{CF}_{\mathrm{MEEY}} \\
\left(\mathrm{kg} \mathrm{CO}_{2} \text {-eq kg }{ }^{-1} \mathrm{yr}^{-1}\right)\end{array}$} & \multirow{2}{*}{$\begin{array}{l}\mathrm{CF}_{\mathrm{E}} \\
\left(\mathrm{kg} \mathrm{CO}_{2}-\mathrm{eq}^{-1} \mathrm{~s}^{-1} \mathrm{yr}^{-1}\right)\end{array}$} \\
\hline & & & & & \\
\hline \multirow[t]{4}{*}{ RT } & MWSW & $2795.52 \mathrm{~g}$ & $7.32 \mathrm{e}$ & $0.19 \mathrm{e}$ & $0.83 \mathrm{~g}$ \\
\hline & SWMW & $3187.37 \mathrm{f}$ & $8.54 \mathrm{de}$ & $0.21 \mathrm{~d}$ & $0.97 \mathrm{f}$ \\
\hline & SW & $4896.87 \mathrm{~b}$ & $13.50 \mathrm{~b}$ & $0.29 \mathrm{c}$ & $1.27 \mathrm{~d}$ \\
\hline & MW & $1991.74 \mathrm{~h}$ & $4.42 \mathrm{f}$ & $0.13 \mathrm{f}$ & $0.58 \mathrm{~h}$ \\
\hline \multirow[t]{4}{*}{ NT } & MWSW & $4792.39 \mathrm{c}$ & $13.26 \mathrm{~b}$ & $0.33 \mathrm{~b}$ & $1.53 b$ \\
\hline & SWMW & 5584.49 a & $17.23 \mathrm{a}$ & $0.43 a$ & $2.05 a$ \\
\hline & SW & $3328.53 \mathrm{e}$ & $11.58 \mathrm{c}$ & $0.24 \mathrm{~d}$ & $1.17 \mathrm{e}$ \\
\hline & MW & $3669.62 \mathrm{~d}$ & $9.92 \mathrm{~d}$ & $0.30 \mathrm{c}$ & $1.43 \mathrm{c}$ \\
\hline \multicolumn{6}{|c|}{$\begin{array}{l}\mathrm{CF}_{\mathrm{A}} \text { : the } \mathrm{CF} \text { per unit of area; } \mathrm{CF}_{\mathrm{EY}} \text {, the } \mathrm{CF} \text { per kg of energy yield; } \mathrm{CF}_{\mathrm{MEEY}} \text {, the } \mathrm{CF} \text { per kg of maize equivalent economic yield; } \mathrm{CF}_{\mathrm{E}} \text {, the } \mathrm{CF}_{\text {per }} \text { unit } \\
\text { of total revenue. RT: rotary tillage; NT: no-till. MWSW: maize-wheat-soybean-wheat; SWMW: soybean-wheat-maize-wheat; SW: soybean- } \\
\text { wheat; and MW: maize-wheat. Different letters in the same column indicate significant differences at } P<0.05 \text {. }\end{array}$} \\
\hline
\end{tabular}

Among CF inventories, the SOC sequestration offset $\mathrm{CF}_{\mathrm{A}}$ (Fig. 4) by 34.8\%, 33.2\%, 23.2\%, and 38.2\% under RT-MWSW, RT-SWMW, RT-SW, and RTMW, respectively. Similarly, the SOC sequestration offset $25.6 \%, 21.1 \%, 31.5 \%$, and $32.0 \%$ of CF $_{A}$ under NT-MWSW, NT-SWMW, NT-SW, and NT$\mathrm{MW}$, respectively. The emission of $\mathrm{N}_{2} \mathrm{O}$ accounted for $23.1-29.1 \%$ of $\mathrm{CF}_{\mathrm{A}}$ among treatments. Among indirect emissions from agricultural inputs, fertilizer accounted for most of $\mathrm{CF}_{\mathrm{A}}$ (Fig. 4), and it ranged from 13.0-16.1\%, irrigation was the secondary contributor and it ranged from 8.1$11.3 \%$, and herbicides contributed the lowest and ranged from $0.03-0.2 \%$.

\subsection{Carbon sustainability index (CSI)}

The highest CSI was observed under RT-MW, which was 105.8\%, 122.3\%, 347.7\%, 281.2\%, 493.0\%, 247.7\%, and 168.3\% higher than that under RT-MWSW, RT-SWMW, RT-SW, NT-MWSW, NT-SWMW, NT-SW, and NT-MW, respectively. The lowest CSI was observed under NT-SWMW. There were no significant differences between RT-MWSW and RT-SWMW.

\subsection{Comprehensive assessment of economic and environmental benefits}

Among the assessed indicators for different CSs under RT, MW has the highest GY and EY due to increased grain yield, as well as the highest CSI and $\triangle$ SOC. SW had the highest MEEY and net revenue. For NT, the highest and lowest GHGs emissions were observed under SWMW and SW, respectively. Furthermore, NT-MWSW has a higher MEEY and net revenue than others. NT-MW had the highest GY, EY, $\triangle$ SOC, and CSI (Fig. 6).

\section{Discussion}

\subsection{Crop yields and economic profits}

The data presented herein indicate that crop yields of wheat, maize, and soybean were significantly affected by tillage and CS treatments, but non-significant interactions were observed between tillage and CSs. This trend was similar to that reported by Hemmat and Eskandari (2004). However, significant interactions between tillage and crop rotation have been reported in crop yield during dry years (Ray et al., 2012; Huynh, et al., 2019). Results of the present study also show that, despite differences among tillage practices, the EY of MW was higher than that of soybean involved in CSs. This trend may be due to the lower photosynthesis and also photorespiration of soybean than those of maize (Zhang and $\mathrm{He}$, 2020). The MEEY of CSs including soybean were higher than that of MW. Therefore, establishing a CA system based on soybean rotation could increase the MEEY, mainly because of the compensatory effects from high price over the low crop yield of soybean. However, 
different results have been reported for previous studies in different regions (Uzoh et al., 2019; Sun et al., 2021). Decreases in crop yield under NT were observed in the present study. Zhang et al. (2013) obtained the lowest yield of NT wheat among the four tillage treatments for a 3-year field study. This trend may be attributed to soil compaction caused by continuous NT, resulting in limited root growth (Kan et al., 2020c) and increased incidence of weeds (Arvidsson et al., 2014).

Similarly, a previous study also reported that a lower input: output economic ratio observed under NT may be due to somewhat lower crop yield. Decline in crop yield under NT may be caused by the reduced number of mechanical operations and agricultural inputs surpassed the costsaving compensation of adopting NT (Peng and Zhang, 2006). It is generally believed that increase diversification of crop rotation system could enhance the agronomic and environmental benefits of NT (Yang et al., 2014). However, the present study leads to the conclusion that the benefit of SW and MW was higher than that of MWSW and SWMW because of the relative lower grain yield of soybean (Canalli et al., 2020). However, soybean-based CA can improve the economics of cropping systems compared to maize because of its higher economic price.

\subsection{SOC storage}

The results presented in this study showed a higher SOC storage under RT than that under NT, and highest SOC storage and sequestration rate were observed under RT-MW. Most of the previous studies indicated an enhancement in SOC storage under conservation tillage (Snyder et al., 2009; Kan et al., 2020a), mainly due to reduced surface disturbance, increased soil cover, and enhanced input of substrates (Sindelar et al., 2015). Debate also exits if SOC is enhanced or merely redistributed within the soil profile by conservation tillage (Powlson et al., 2014). Some studies have suggested that NT increased SOC in surface soils, but RT (or plow tillage) increased SOC in deeper soils (Lal, 1997; Blanco-Canqui and Lal, 2008). The data from the present study verifies the results of previous studies that increase in SOC in subsoil is also essential to an objective assessment on SOC storage (Huggins et al., 2007). The results presented herein indicate that SOC sequestration under RT was higher than that under NT when considering storage in the subsoil. The reason of the lower SOC storage and sequestration under NT may be the reduced input of total substrates (relatively lower crop yield) from aboveground biomass input, roots, root exudates, etc.

The SOC content also differed significantly among CS treatments. Soybean-based crop rotation strongly impacted the accumulation of SOC. The highest SOC sequestration in the presented study was observed in WM. Similar results were reported in a previous study in the NCP (Shen et al., 2018). The SOC sequestration of MW was slightly higher than that of SW. Results from present study also show that soybean-based crop rotation may reduce the SOC sequestration capacity of CA when compared to maize in NCP. However, most of the previous studies reported that: decomposition of crop residues could be accelerated by soybean compared to maize, and thus increase SOC content (Huggins et al., 2007; Schmer et al., 2020). The differences may be induced by the reduction of C input (King and Blesh, 2018) and the short experimental duration (Shrestha et al., 2013). Thus, optimizing the field operations and continuous use of NT for a longer duration may offset the negative impacts of soybean-based crop rotation on SOC in CA system.

\subsection{Carbon footprints}

$\mathrm{CF}$ is defined as the sum of $\mathrm{GHG}$ emissions and elimination over the whole production process expressed in $\mathrm{CO}_{2}$-eq based on life cycle assessment using a single impact category (ISO, 2013). Different functional units (e.g., $\mathrm{CF}_{\mathrm{EY}}, \mathrm{CF}_{\mathrm{MEEY}}$, and $\mathrm{CF}_{\mathrm{E}}$ ) were used to show the trade-offs between GHGs emissions and other ecosystem services (Sun et al., 2021). Liu et al. (2016) reported that reducing tillage intensity and increasing crop diversity can effectively decline the CF of crop production and increase SOC sequestration. But several studies have reported the opposite results (Lal, 2003; Gregorich et al., 2005; Blanco-Canqui and Lal, 2008). Among the eight treatments in the present study, the minimum and maximum values of CF were observed under RT-MW and NT-SWMW, respectively. The consistent results for the three $\mathrm{CF}_{\text {indicators }}\left(\mathrm{CF}_{\mathrm{EY}}, \mathrm{CF}_{\mathrm{MEEY}}\right.$ and $\mathrm{CF}_{\mathrm{E}}$ ) leads to conclusion that $\mathrm{CF}$ of $\mathrm{RT}$ was lower than that under $\mathrm{NT}$, and soybean-based crop rotation could effectively reduce the $\mathrm{CF}_{\mathrm{E}}$ in the CA system even with a relative low yield of soybean.

Among CF inventories and components, SOC sequestration made the largest contribution to offset GHG emissions, which did not increase with the increase in crop diversity under NT. This trend was mainly because of the limitation of the short experiment duration for crop rotation. A previous study concluded that SOC dynamics along with duration of experiment and the robust results need a long-term assessment (Wang et al., 2021). Combining the tillage and CSs, the lowest CF was observed under RT-MW even with the highest consumption of fertilizer and fuel (i.e., the indirect GHGs emission). Such a trend may be attributed to higher yield and SOC sequestration under RT-MW (Yang et al., 2017). CA with soybean-based crop rotation, as shown in the present study may reduce farming inputs (the indirectly GHGs emission) and increase economic profit, but enhance the risks of decrease in crop yield.

\subsection{Limitations, implications, and perspectives}

The present study assessed the trade-offs between crop productivity and environmental impacts of NT with soybean-based crop rotation in CA system using multiple CF indicators. Among several limitations in data collection and analysis is the fact that $\mathrm{N}_{2} \mathrm{O}$ emissions and the emission factor were directly and indirectly affected by $\mathrm{N}$ application rates and environmental factors (Castanheira and Freire, 2013). $\mathrm{N}_{2} \mathrm{O}$ emission of different planting systems was compared in this study. However, this study did not consider the changes in $\mathrm{N}_{2} \mathrm{O}$ emission factors under different 
precipitation conditions, which may affect the accuracy of CF calculation. In addition, some other gas emissions (i.e., nitric oxide, C monoxide, and ammonia) also affected some complex atmospheric reactions (Monson and Holland, 2001), and these were not calculated. This trend was mainly because the effect on the $\mathrm{C}$ budget was generally too small to have a significant impact on the results. Furthermore, due to the limitation of the small area study, the calculation of the manufacture, storage, and transportation of agricultural inputs was not accurate, and the price of farm products also fluctuated between years. This limitation affected the absolute values of CF under different treatments. The comparisons and relative changes among treatments were creditable. Therefore, this study provides a useful reference for the comprehensive economic and environmental assessment of CA in the NCP.

Previous studies have predicted that global demand for food will double over the next 50 years, posing significant challenges to environmental sustainability and increasing food supply without compromising environmental hazards (Tilman et al., 2002). There have been many studies on how CA improves crop yield and ecosystem services, but few studies on how legume crops will interact with other CA principles (e.g., NT or RR) on the environmental footprint. Conventional agriculture requires excessive amounts of chemicals to boost crop productivity. However, the combination of legume crops in CA can reduce the application of $\mathrm{N}$ fertilizer due to its symbiotic nitrogen fixation. We promoted CA (including $\mathrm{NT}$, soybean-based crop rotation, straw mulching) with the best agronomic measures to meet the environmental challenges trying to establish a suitable case.

The analysis presented herein show that combination of soybean-based crop rotation with NT offers great potential to improve the economic and environmental efficiency in the NCP. It will promote the regional sustainable agriculture development. The present study has revealed a future potential to sustain food supply and enhance crop diversity by increasing soybean yield in CA system with the aim to increase the agricultural sustainability. Future research priorities include in-depth studies on how to realize the potential crop yield under CA system under on farm conditions.

\section{Conclusions}

The results presented support the following conclusions:

1. Despite the risks of declining grain yield in NT (i.e., average of $14.4 \%$ lower than that under RT), the fewer inputs and the combined benefits from soybean-based crop rotation could balance the trade-offs with environmental and economic profits

2. $\mathrm{N}_{2} \mathrm{O}$ emission was the main contributor to $\mathrm{CF}$, indicating a high potential to reduce GHGs by optimizing fertilizer application and related field management practices.

3. In combination with soybean-based crop rotation, CA could reduce CF due to less input of $\mathrm{N}$ fertilizer and more $\mathrm{N}$ fixation.

4. The lower yield under NT partly offsets its environmental benefits.

5. The long-term economic and environmental sustainability of agricultural production in the NCP can be improved by establishing a CA system that combines straw return as mulch with suitable crop diversity (especially leguminous crops).

\section{Declarations}

\section{Authors Contributions}

Conceptualization, Data curation, and writing - Original draft preparation.: Wen-Xuan Liu;

Investigation: Wen-Sheng Liu, Mu-Yu Yang, Yu-Xin Wei, Zhe Chen, Ahmad Latif Virk;

Writing- Reviewing and Editing: Rattan Lal;

Formal analysis: Xin Zhao;

Project administration and supervision: Hai-Lin Zhang.

\section{Funding}

This work was supported by the National Natural Science Foundation of China (32001486).

\section{Data availability}

Data are available within the article. The authors confirm that the data supporting the findings of this study are available within the article. 
Ethics approval and consent to participate: Not applicable.

Consent for publication: Not applicable.

Competing interests: The authors declare no competing interests.

\section{References}

1. Ali SA, Tedone L, Verdini L, De Mastro G (2017) Effect of different crop management systems on rainfed durum wheat greenhouse gas emissions and carbon footprint under Mediterranean conditions. J Clean Prod 140:608-621

2. Arvidsson J, Etana A, Rydberg T (2014) Crop yield in Swedish experiments with shallow tillage and no-tillage 1983-2012. Eur J Agron 52:307-315

3. Bao S (2008) Soil Agrochemical Analysis. China Agriculture Press Beijing. (in Chinese).

4. Blanco-Canqui H, Lal R (2008) No-Tillage and Soil-Profile Carbon Sequestration: An On-Farm Assessment. Soil Sci Soc Am J 72:693-701

5. Canalli LBD, da Costa GV, Volsi B, Leocádio ALM, Neves CSVJ, Telles TS (2020) Production and profitability of crop rotation systems in southern Brazil. Semina-Ciên Agrár 41:2541-2541

6. Castanheira ÉG, Freire F (2013) Greenhouse gas assessment of soybean production: implications of land use change and different cultivation systems. J Clean Prod 54:49-60

7. Chen F (2002) Agroecology. China Agricultural University Press, Beijing. (in Chinese).

8. Chen S, Lu F, Wang X (2015) Estimation of greenhouse gases emission factors for China's nitrogen, phosphate, and potash fertilizers. Acta Ecol Sin 35:6371-6383. (in Chinese).

9. Ellert BH, Bettany JR (1995) Calculation of organic matter and nutrients stored in soils under contrasting management regimes. Ca J Soil Sci 75:529-538

10. Fang JY, Guo ZD, Piao SL, Chen AP (2007) Terrestrial vegetation carbon sinks in China, 1981-2000. Sci China Ser D 50:1341-1350

11. Gregorich E, Rochette P, Vandenbygaart A, Angers D (2005) Greenhouse gas contributions of agricultural soils and potential mitigation practices in Eastern Canada. Soil Till Res 83:53-72

12. Guo J (2015) Research progress on the impact of climate change on China's agricultural production. J Appl Meteorol 26:1-11. (in Chinese).

13. Halvorson AD, Del Grosso SJ, Reule CA (2008) Nitrogen, tillage, and crop rotation effects on nitrous oxide emissions from irrigated cropping systems. J Environ Qual 37:1337-1344

14. Hemmat A, Eskandari I (2004) Tillage system effects upon productivity of a dryland winter wheat-chickpea rotation in the northwestd region of Iran. Soil Till Res 78:69-81

15. Huang MB, Shao MG, Zhang L, Li YS (2003) Water use efficiency and sustainability of different long-term crop rotation systems in the Loess Plateau of China. Soil Till Res 72:95-104

16. Huggins DR, Allmaras RR, Clapp CE, Lamb JA, Randall GW (2007) Corn-Soybean Sequence and Tillage Effects on Soil Carbon Dynamics and Storage. Soil Sci Soc Am J 71:238-244

17. Huynh HT, Hufnagel J, Wurbs A, Bellingrath-Kimura SD (2019) Influences of soil tillage, irrigation and crop rotation on maize biomass yield in a 9-year field study in Müncheberg, Germany. Field Crop Res 241:107565

18. IPCC (2006) Agriculture, forestry, and other land use. IPCC guidelines for national greenhouse gas inventories. Institute for Global Environmental Strategies, Paris

19. IPCC (2013) Climate Change 2013: the Physical Science Basis. Cambridge University Press, Cambridge

20. IPCC (2018) Global warming of $1.5^{\circ} \mathrm{C}$. World Meteorological Organization, Geneva, Switzerland

21. IS014067 (2013) Greenhouse Gases-Carbon Footprint of Products-Requirements and Guidelines for Quantification and Communication. International Organization for Standardization, Geneva, Switzerland

22. Janzen HH, Angers DA, Boeh M, Bolinder M, Desjardins RL, Dyer JA, Ellert BH, Gibb DJ, Gregorich EG, Helgason BL, Lemke R, Massé $D$, McGinn SM, McAllister TA, Newlands N, Pattey E, Rochette P, Smith W, VandenBygaart AJ, Wang H (2006) A proposed approach to estimate and reduce net greenhouse gas emissions from whole farms. Can J Soil Sci 86:401-418

23. Kan Z, Virk AL, Wu G, Qi J, Ma S, Wang X, Zhao X, Lal R, Zhang H (2020a) Priming effect intensity of soil organic carbon mineralization under no-till and residue retention. Appl Soil Ecol 147:103445

24. Kan Z, Ma S, Liu Q, Liu B, Virk AL, Qi J, Zhao X, Lal R, Zhang H (2020b) Carbon sequestration and mineralization in soil aggregates under long-term conservation tillage in the North China Plain. CATENA 188:104428

25. Kan Z, Liu Q, He C, Jing Z, Virk AL, Qi J, Zhao X, Zhang H (2020c) Responses of grain yield and water use efficiency of winter wheat to tillage in the North China Plain. Field Crop Res 249:107760

Page $12 / 17$ 
26. King AE, Blesh J (2018) Crop rotations for increased soil carbon: perenniality as a guiding principle. Ecol Appl 28:249-261

27. Ladha JK, Rao AN, Raman AK, Padre AT, Dobermann A, Gathala M, Kumar V, Saharawat Y, Sharma S, Piepho HP, Alam MM, Liak R, Rajendran R, Reddy CK, Parsad R, Sharma PC, Singh SS, Saha A, Noor S (2016) Agronomic improvements can make future cereal systems in South Asia far more productive and result in a lower environmental footprint. Glob Change Biol 22:1054-1074

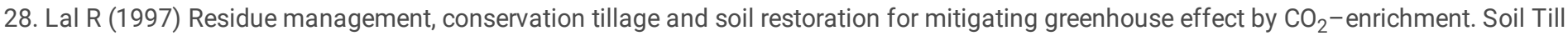
Res 43:81-107

29. Lal R (2003) Global Potential of Soil Carbon Sequestration to Mitigate the Greenhouse Effect. Crit Rev Plant Sci 22:151-184

30. Lal R (2004) Carbon emission from farm operations. Environ Int 30:981-990

31. Lal R (2015) A system approach to conservation agriculture. J Soil Water Conserv 70:82A-88A

32. Li H, Qiu J, Wang L, Tang H, Li C, Van Ranst E (2010) Modelling impacts of alternative farming management practices on greenhouse gas emissions from a winter wheat-maize rotation system in China. Agr Ecosyst Environ 135:24-33

33. Liang Q, Chen H, Gong Y, Fan M, Yang H, Lal R, Kuzyakov Y (2011) Effects of 15 years of manure and inorganic fertilizers on soil organic carbon fractions in a wheat-maize system in the North China Plain. Nutr Cycl Agroecosys 92:21-33

34. Liu C, Cutforth H, Chai Q, Gan Y (2016) Farming tactics to reduce the carbon footprint of crop cultivation in semiarid areas. A review. Agron Sustain Dev 36:69

35. Liu Q, Xu C, Han S, Li X, Kan Z, Zhao X, Zhang H (2021) Strategic tillage achieves lower carbon footprints with higher carbon accumulation and grain yield in a wheat-maize cropping system. Sci Total Environ 798:149220

36. Lötjönen S, Ollikainen M (2018) Does crop rotation with legumes provide an efficient means to reduce nutrient loads and GHG emissions? Rev Agric Food Environ Stud 98:283-312

37. Meng T (2020) Effects of Tillage Methods on Soil Organic Carbon and Total Nitrogen Content in the Loess Plateau. Asian Agric Res 12:2527

38. Monson RK, Holland EA (2001) Biospheric trace gas fluxes and their control over troposphericchemistry.Annu Rev Ecol Syst32:547-576

39. NDRC (2011) Provincial Greenhouse Gas List Preparation Guidelines. The People's Republic of China

40. Peng W, Zhang Y (2006) Agricultural Research in the Arid Areas. 24: 113-118 (in Chinese)

41. Pradhan A, Chan C, Roul PK, Halbrendt J, Sipes B (2018) Potential of conservation agriculture (CA) for climate change adaptation and food security under rainfed uplands of India: a transdisciplinary approach. Agr Syst 163:27-35

42. Powlson DS, Stirling CM, Jat ML, Gerard BG, Palm CA, Sanchez PA, Cassman KG (2014) Limited potential of no-till agriculture for climate change mitigation. Nature Climate Change 4:678-683

43. Ray DK, Ramankutty N, Mueller ND, West PC, Foley JA (2012) Recent patterns of crop yield growth and stagnation. Nat Commun 3:1293

44. Ray M, Roy DC, Zaman A (2016) Evaluation of rice (Oryza sativa)-based cropping systems for increasing productivity, resource-use efficiency and energy productivity in coastal West Bengal. Indian J Agron 61:131-137

45. Schenck R, Huizenga D (2014) Proceedings of the 9th international conference on life cycle assessment in the Agri- Food Sector (LCA Food 2014). San Francisco, California, USA, 8-10 October, 2014

46. Schmer MR, Jin VL, Wienhold BJ, Becker SM, Varvel GE (2020) Long-term rotation diversity and nitrogen effects on soil organic carbon and nitrogen stocks. Agrosyst Geosci Environ 3:e20055

47. Shen Y, Sui P, Huang J, Wang D, Whalen J, Chen Y (2018) Global warming potential from maize and maize-soybean as affected by nitrogen fertilizer and cropping practices in the North China Plain. Field Crop Res 225:117-127

48. Shrestha BM, McConkey BG, Smith WN, Desjardins RL, Campbell CA, Grant BB, Miller PR (2013) Effects of crop rotation, crop type and tillage on soil organic carbon in a semiarid climate. Can J Soil Sci 93:137-146

49. Sindelar AJ, Lamb JA, Coulter JA (2015) Short-Term Stover, Tillage, and Nitrogen Management Affect Near-Surface Soil Organic Matter. Soil Sci Soc Am J 79:251-260

50. Snyder CS, Bruulsema TW, Jensen TL, Fixen PE (2009) Review of greenhouse gas emissions from crop production systems and fertilizer management effects. Agr Ecosyst Environ 133:247-266

51. Sun T, Feng X, Lal R, Cao T, Guo J, Deng A, Zheng C, Zhang J, Song Z, Zhang W (2021) Crop diversification practice faces a tradeoff between increasing productivity and reducing carbon footprints. Agr Ecosyst Environ 321:107614

52. Tilman D, Cassman K, Matson P, Naylor R, Polasky S (2002) Agricultural sustainability and intensive production practices. Nature 418:671677

53. Uzoh I, Igwe CA, Okebalama CB, Babalola $O 0$ (2019) Legume-maize rotation effect on maize productivity and soil fertility parameters under selected agronomic practices in a sandy loam soil. Sci Rep-UK 9:8539 
54. van der Werf HMG, Garnett T, Corson MS, Hayashi K, Huisingh D, Cederberg C (2014) Towards eco-efficient agriculture and food systems: theory, praxis and future challenges. J Clean Prod 73:1-9

55. Virk AL, Liu W, Niu J, Xu C, Liu Q, Kan Z, Zhao X, Zhang H (2021) Effects of Diversified Cropping Sequences and Tillage Practices on Soil Organic Carbon, Nitrogen, and Associated Fractions in the North China Plain. J Soil Sci Plant Nut 21:1201-1212

56. Wang P, Chen J, Wang G, Hu J, Li S, Wei L (2018) Comprehensive evaluation of dryland farming rotation systems in Loess Hilly and Gully region. Bull Soil Water Conserv 38:229-234. +240 (in Chinese).

57. Wang X, Jing Z, He C, Liu Q, Qi J, Zhao X, Xiao X, Zhang H (2021) Temporal variation of SOC storage and crop yield and its relationship - A fourteen year field trial about tillage practices in a double paddy cropping system. China Sci Total Environ 759:143494

58. West TO, Marland G (2002) A synthesis of carbon sequestration, carbon emissions, and net carbon flux in agriculture: comparing tillage practices in the United States. Agr Ecosyst Environ 66:1930-1946

59. Yang R, Su Y, Kong J (2017) Effect of tillage, cropping, and mulching pattern on crop yield, soil $\mathrm{C}$ and $\mathrm{N}$ accumulation, and carbon footprint in a desert oasis farmland. Soil Sci Plant Nutr 63:599-606

60. Yang X, Gao W, Zhang M, Chen Y, Sui P (2014) Reducing agricultural carbon footprint through diversified crop rotation systems in the North China plain. J Clean Prod 76:131-139

61. Zeng Z (2018) Research progress and prospect of legumes and gramineae crop rotation. Chinese J Eco-agric 26:57-61. (in Chinese).

62. Zhang J, Sui P, Chen Y, Gao W, Nie Z, Shi L, Xiong J, Jiang H (2013) Crop yield response to different tillage modes combined with fertilization measures. Chinese $\mathrm{J}$ Ecol 32:2721-2727. (in Chinese).

63. Zhang H, Lal R, Zhao X, Xue J, Chen F (2014) Opportunities and challenges of soil carbon sequestration by conservation agriculture in China. Adv Agron 124:1-36

64. Zhang L, He X (2020) Nitrogen utilization mechanism in C3 and C4 plants. Chin Bull Bot 55:228-239

65. Zhang L, Bian X, Liu Y (2001) Studies on water consumption characteristics and effects of crops rotation in Plateau of North Hebei Province. Sci Agric Sinica 34:56-60. (in Chinese).

66. Zhang M, Wang F, Chen F, Malemela M, Zhang H (2013) Comparison of three tillage systems in the wheat-maize system on carbon sequestration in the North China Plain. J Clean Prod 54:101-107

67. Zhao X, Virk AL, Ma S, Kan Z, Qi J, Pu C, Yang X, Zhang H (2020) Dynamics in soil organic carbon of wheat-maize dominant cropping system in the North China plain under tillage and residue management. J Environ Manage 265:110549

68. Zhao Y, Wang M, Hu S, Zhang X, Ouyang Z, Zhang G, Huang B, Zhao S, Wu J, Xie D, Zhu B, Yu D, Pan X, Xu S, Shi X (2018) Economics- and policy-driven organic carbon input enhancement dominates soil organic carbon accumulation in Chinese croplands. P Natl Acad Sci USA 115:4045-4050

69. Zhu B, Cheng W (2012) Nodulated soybean enhances rhizosphere priming effects on soil organic matter decomposition more than nonnodulated soybean. Soil Bio Biochem 51:56-65

\section{Figures}

MWSW

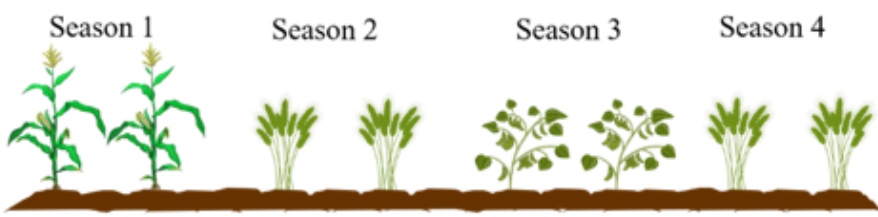

SWMW

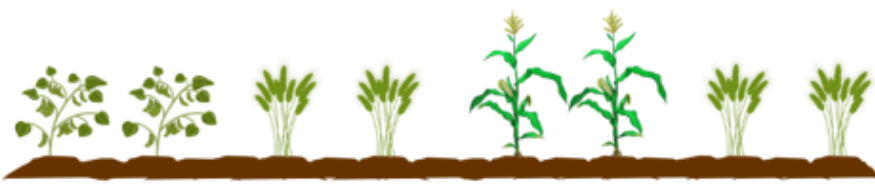

SW

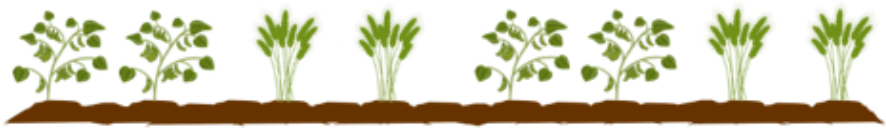

MW

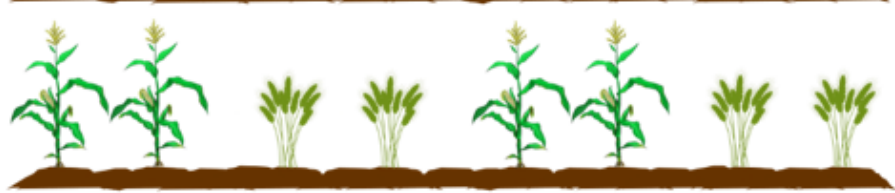

Page $14 / 17$ 
Figure 1

Schematic diagram of the different cropping sequences from October 2018 to June 2020 in the present study. MWSW: maize-wheat-soybeanwheat; SWMW: soybean-wheat-maize-wheat; SW: soybean-wheat; and MW: maize-wheat.

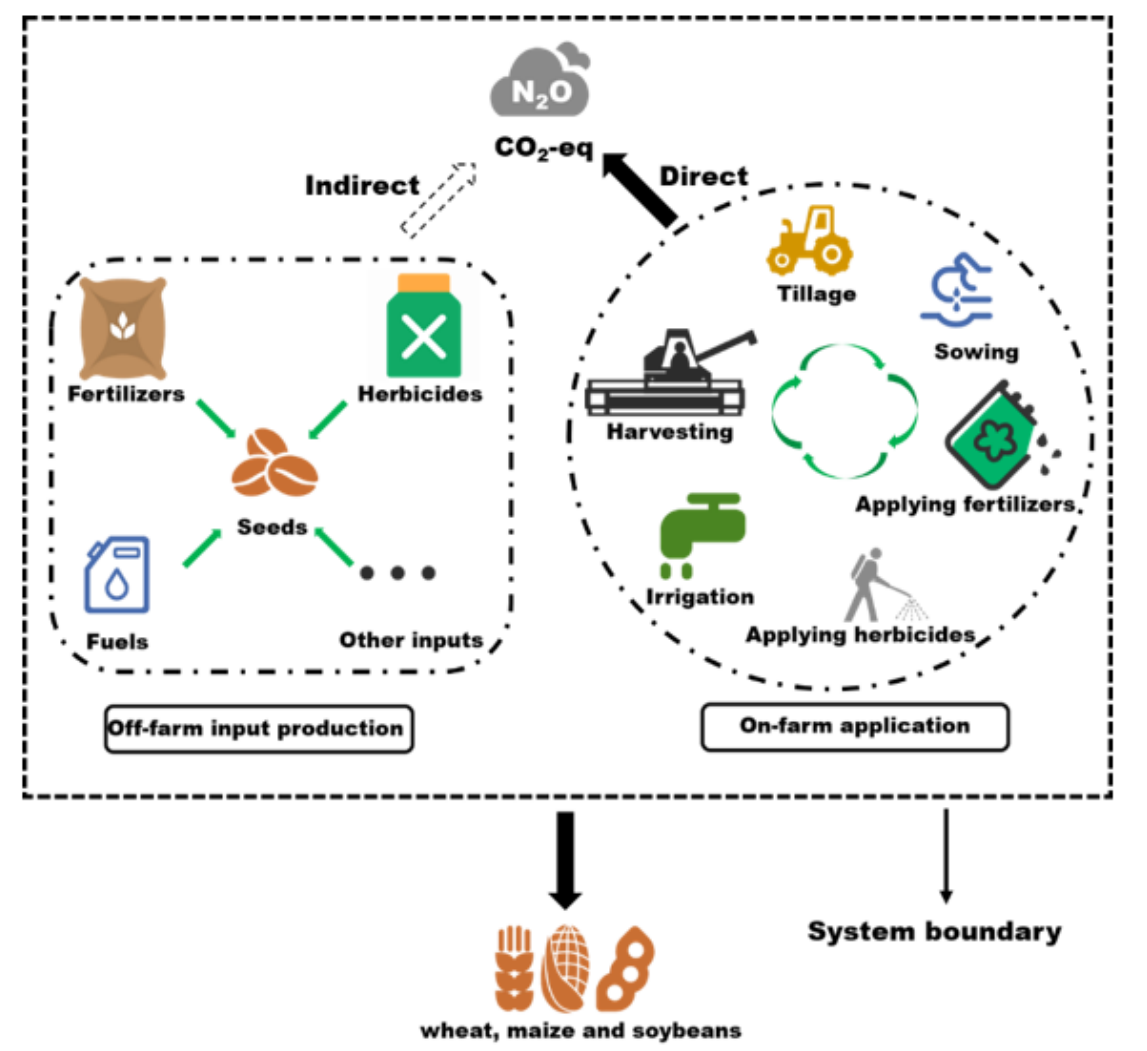

Figure 2

System boundary for calculating GHGs emissions in different tillage practices and cropping sequences.
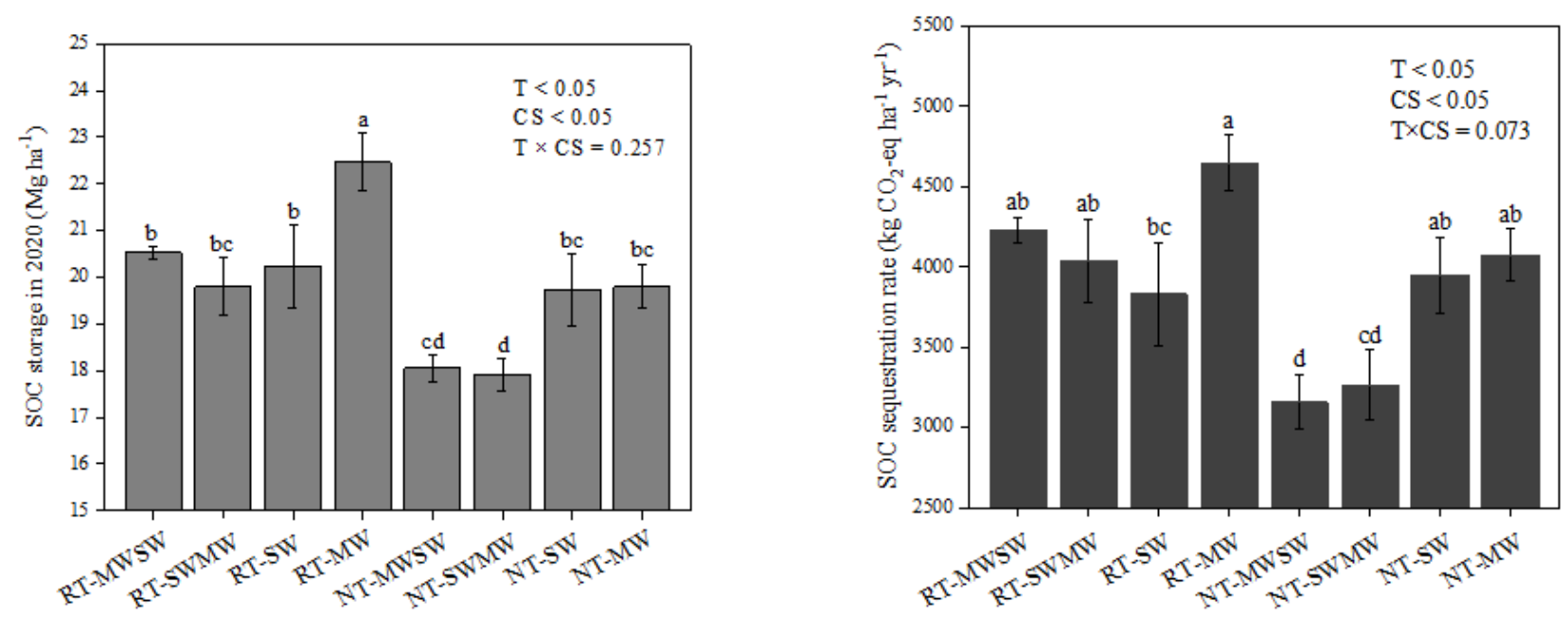

Figure 3

SOC storage in 2020 and SOC sequestration rate under different tillage practices and cropping sequences. T, tillage practices; CS, cropping sequences. RT, rotary tillage; NT, no-till. MWSW, maize-wheat-soybean-wheat; SWMW, soybean-wheat-maize-wheat; SW, soybean-wheat; and MW, maize-wheat. Different lowercase represents significant difference at $P<0.05$ between treatments. 


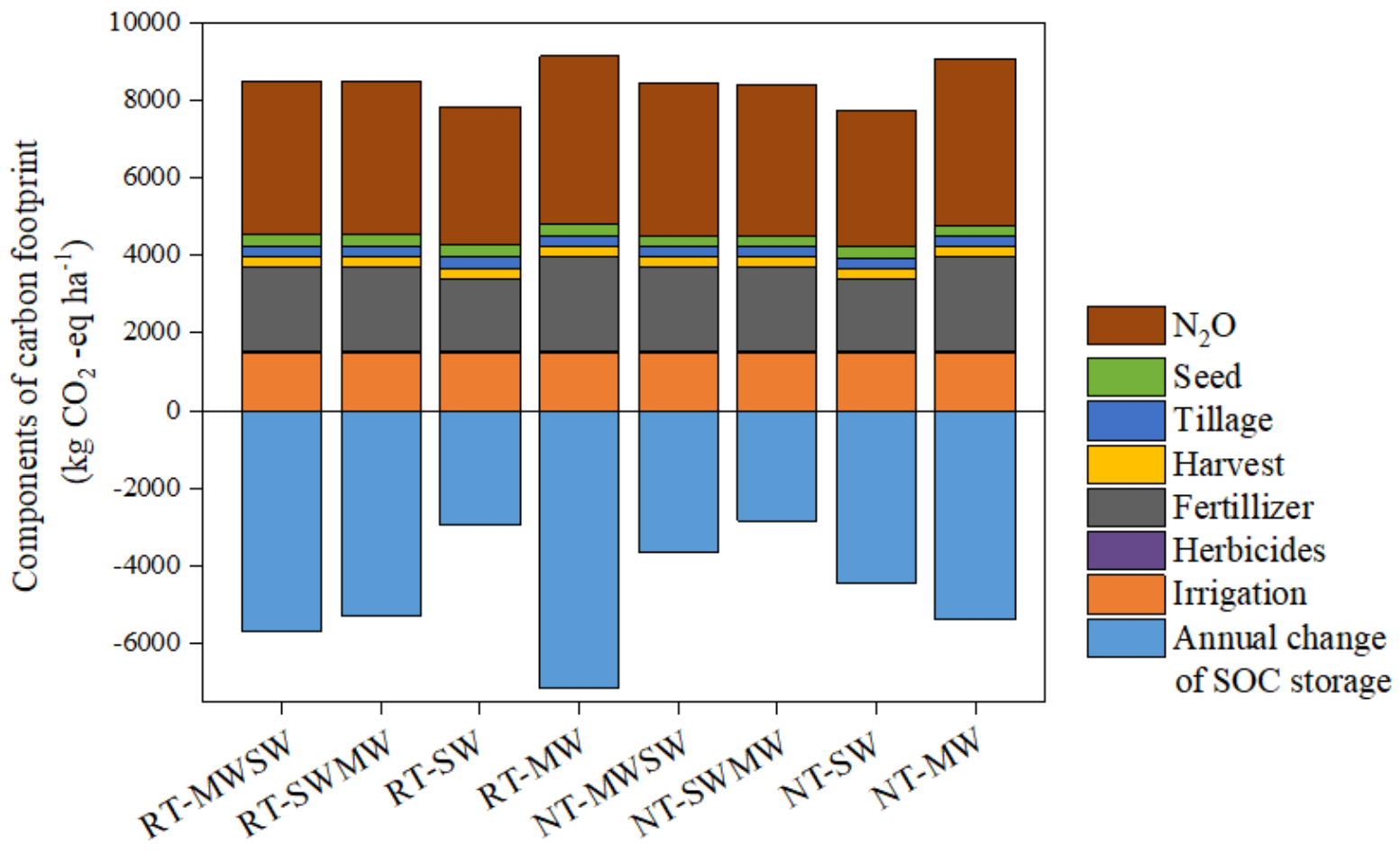

Figure 4

Component of carbon footprint of the entire growing season under different tillage practices and cropping sequences. RT, rotary tillage; NT, notill. MWSW, maize-wheat-soybean-wheat; SWMW, soybean-wheat-maize-wheat; SW, soybean-wheat; and MW, maize-wheat.

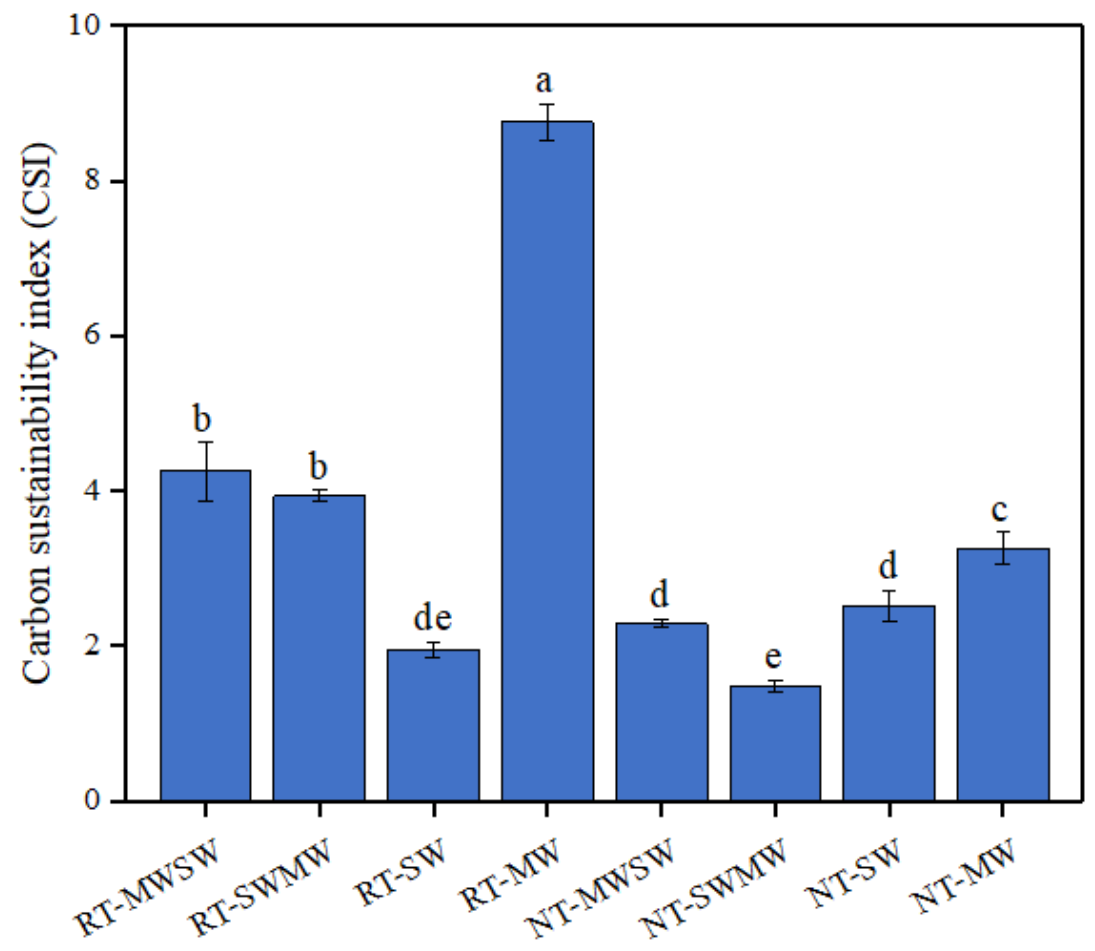

Figure 5

Carbon sustainability index (CSI) of different tillage practices and cropping sequences. RT, rotary tillage; NT, no-till. MWSW, maize-wheatsoybean-wheat; SWMW, soybean-wheat-maize-wheat; SW, soybean-wheat; and MW, maize-wheat. Different lowercase represents significant difference at $P<0.05$ between treatments. 

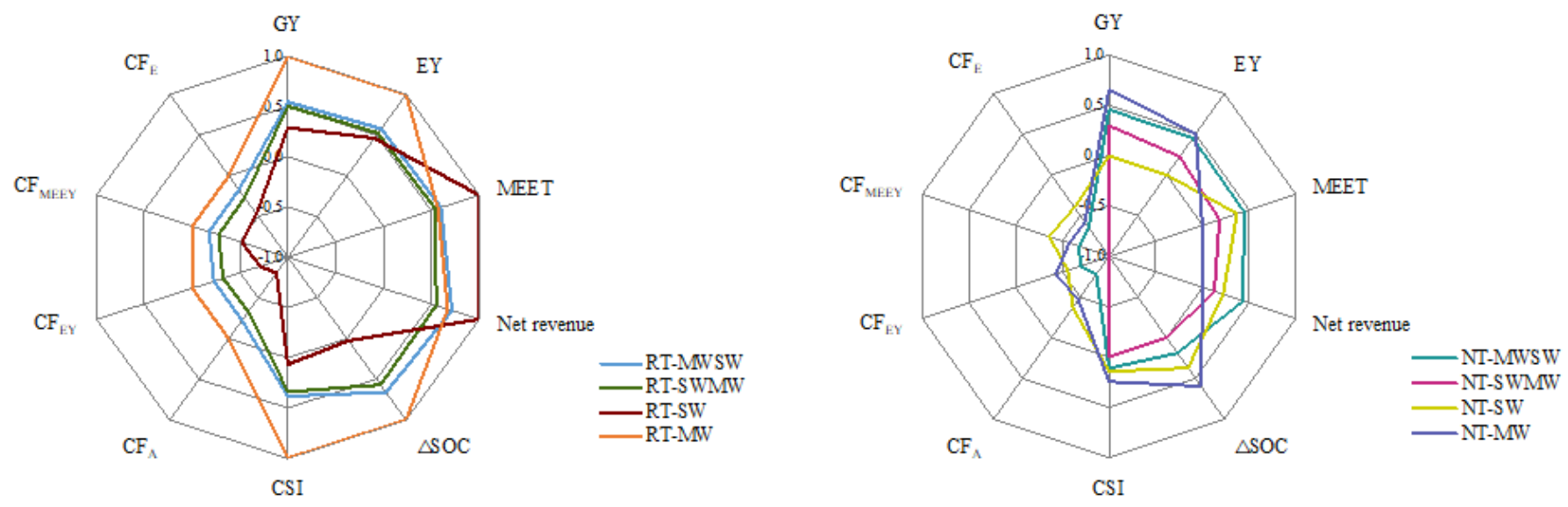

\section{Figure 6}

Performance of selected parameters for different tillage practices and cropping sequences using radar chart. GY, grain yield; EY, energy yield; MEEY, maize equivalent economic yield; $\triangle S O C$, the mean annual $S O C$ change in $0-30 \mathrm{~cm}$ soil depth; $C S I$, carbon sustainability index; $C F_{A}$, the $\mathrm{CF}$ per unit of area; $\mathrm{CF}_{\mathrm{EY}}$, the $\mathrm{CF}$ per $\mathrm{kg}$ of energy yield; $\mathrm{CF}_{\mathrm{MEEY}}$, the $\mathrm{CF}$ per $\mathrm{kg}$ of maize equivalent economic yield; $\mathrm{CF}_{\mathrm{E}}$, the $\mathrm{CF}$ per unit of total revenue. RT, rotary tillage; NT, no-till. MWSW, maize-wheat-soybean-wheat; SWMW, soybean-wheat-maize-wheat; SW, soybean-wheat; and MW, maize-wheat. 